\title{
Next Generation PONs with Wireless Backhauling
}

\author{
Milos Milosavljevic, Pandelis Kourtessis, Member, IEEE, Wansu Lim, John M. Senior \\ Optical Networks Group, Science and Technology Research Institute (STRI), University of Hertfordshire \\ College Lane Campus, Hatfield, AL10 9AB, UK \\ Tel: +44 (0) 1707286279, e-mail: m.milosavljevic@herts.ac.uk
}

\begin{abstract}
This paper is tackling the issue that stem from the coexistence of diverse technologies, such as emerging OFDMA-PON and WiMAX/LTE, within the same infrastructure. It outlines the key requirements that need to be fulfilled for the backhauling links supporting next generation wireless standards. These links should provide long reach as well as low-cost remote radio base stations coupled with centralised processing. To that extent, the industrial standard simulation platforms were used to evaluate the key network features from the PHY perspective gained by the convergence. The network modelling results confirm the transmission of $16 \mathrm{CPRI}$ signals up to $100 \mathrm{~km}$ OFDMA-PON infrastructures achieving $40 \mathrm{Gbit} / \mathrm{s}$ total aggregate data rates.
\end{abstract}

Keywords: OFDMA-PON, wireless backhauling, CPRI, LTE/WiMAX, Digital over Fibre (DoF).

\section{INTRODUCTION}

With the developments in broadband wireless networks, driven primarily by WiMAX [1] and LTE [2], data rates in the range of hundreds of Mbits are expected to be supported over next generation radio cells. This will unavoidably lead to the deployment of densely populated base stations with high spectral efficiency. In addition inter-site distances will be significantly reduced while the number of remote radio nodes increased. High bandwidth, cost-effective backhauling links would be required as a result to connect each of these base stations to a common central office (CO). Since the application of current solutions, such as SDH and IP is anticipated expensive to both deploy and operate, lower-cost optical infrastructures have been suggested, required also to allow sharing of network resources among different operators.

In addition, the common public radio interface (CPRI) specifications are standardised [3] to provide a cost effective solution for service providers to support emerging broadband wireless topologies since simple remote radio base stations could be located at environmentally challenging locations and controlled centrally from the head office. As the aggregate data rates conveyed over these links are expected to be in the range of $2.5 \mathrm{Gbit} / \mathrm{s}$, or more, for $20 \mathrm{MHz}$ LTE channels per base-station (BS), high bandwidth optical backhauling links need to be developed to meet the required demand at converged ONU/BSs. Orthogonal frequency division multiple access passive optical networks (OFDMA-PONs) are emerging as high bandwidth, long-reach solutions for the aggregation of various services on a single platform $[4,5]$. To that extent, this paper presents a VPI/MATLAB network model demonstrating the transmission of 16 CPRI wireless signal formats over an OFDMA-PON infrastructure [4], exhibiting $40 \mathrm{Gbit} / \mathrm{s}$ aggregate data rates and up to $100 \mathrm{~km}$ fiber spans.

\section{REQUIREMENTS FOR WIRELESS BACKHAULING}

Concerning the requirements on fixed access networks originating from mobile radio networks the main trend that could be observed is the evolution towards more powerful mobile radio network technologies based on current - or enhanced - network concepts in particular maintaining the role of base stations in the radio access network (RAN). The outcome of this evolution is higher transmission capacities with the target to provide bit rates in order to be able to meet forecasted future mobile radio traffic demands. To that extent, for the emerging LTE standard [6] the estimated peak data rates for the backhaul link are summarized in Table 1 for time division duplex (TDD) frame. This is expected to be increased with the developments of LTE-Advance (LTE-A) requiring backhauling links in the range of $1 \mathrm{Gbit} / \mathrm{s}$ [7].

Table 1. Peak rates for E-UTRA TDD [6].

\begin{tabular}{|l|c|c|c|c|}
\hline & \multicolumn{2}{|c|}{ Downlink } & \multicolumn{2}{c|}{ Uplink } \\
\hline Assumptions & \multicolumn{2}{|c|}{ 64 QAM } & \multicolumn{2}{c|}{ Single TX UE, 64 QAM } \\
\hline Unit & Mbps in 20 MHz & b/s/Hz & Mbps in 20 MHz & b/s/Hz \\
\hline Requirement & 100 & 5.0 & 50 & 2.5 \\
\hline 2x2 MIMO in DL & 142 & 7.1 & 62.7 & \multirow{2}{*}{3.1} \\
\hline 4
\end{tabular}

Another trend which can be observed in mobile radio networks, targets at dividing the conventional base stations into central base stations (CBS) and simple remote antenna units (RAU). The base station is separated into one or several Radio Frequency (RF) transceiver and power amplifier parts (often referred to as remote radio 
units) and one radio controller/baseband part. Benefits for an architecture with centralized processing are expected to be manifold: Compact remote radio head solutions and as a result sites that are simpler to house, install and operate and prospective lower energy consumption. Also the central resource administration and allocation is expected to be beneficial for traffic control.

Therefore, one of the possible options to achieve this target is relaying on recently standardised CPRI signal formats [3]. The CPRI variant is clearly allowing deployment of distributed base station antennas with high flexibility and low deployment cost. With a clear focus on layer 1 and layer 2 the scope of the CPRI specification is restricted to the link interface only, which is basically a point to point interface. Such a link shall have all the features necessary to enable a simple and robust usage of any topology, including a direct interconnection of multiport remote base stations.

Significantly, the CPRI does not define the PHY topology between the central office and remote radio heads as long as certain conditions are satisfied. One of the critical conditions, considered in this investigation as well, is that the BER ratio should be in the range of $1 \mathrm{E}-12$ at the remote antennas. The forward error correction is optional and could be applied if the signal quality cannot satisfy the specified requirements [3].

Following this trend however, the backhauling links must satisfy stringent latency requirements imposed on wireless link due to the centralised processing and long fibre distances. The Table 2 outlines some of the delay figures defined by the standard [6].

Table 2. Latency requirements for E-UTRA [6].

\begin{tabular}{|l|c|}
\hline Parameter & Value \\
\hline Synchronous HARQ & $8 \mathrm{~ms}$ \\
\hline User plane (unload condition) & $5 \mathrm{~ms}$ \\
\hline Control plane & $100 \mathrm{~ms}$ \\
\hline
\end{tabular}

Finally, the next section presents analysis of CPRI transmission over the next generation OFDMA-PON [4] in order to establish the maximum transmission distance and evaluate key network impairments. The latency requirements of the proposed architecture are out of the scope of this paper.

\section{CPRI OVER OFDMA-PON}

As demonstrated by the proposed architecture in Fig. 1, the $2.5 \mathrm{Gbit} / \mathrm{s}$ CPRI data stream [3], assuming $20 \mathrm{MHz}$ of LTE bandwidth, is generated at the central office $(\mathrm{CO})$ and mapped to the optical OFDMA subcarriers. Several BSs could be multiplexed on a single PON depending on the total data rate on the PON and maximum number of supported subcarriers. In order to ensure reliable communication for wireless users, dedicated subcarriers need to be allocated for each BS since the CPRI link requires constant data stream. A significant challenge with this approach is non-linearity of the optical modulators and fibre chromatic dispersion that can significantly degrade the received signal quality at the remote base stations. In addition, the choice of digital to analog and analog to digital converters (DAC and ADC) resolution largely influence the performance of the proposed system and it usually constrains practical implementation.

According to the deployed scenario shown in Fig. 1, 16-QAM modulation for each subcarrier was assumed with 8, 9 and 10 bits ADC/DAC resolution. Therefore, with $40 \mathrm{Gbit} / \mathrm{s}$ total aggregate data rate and 256 subcarriers the maximum rate per subcarrier is $156.25 \mathrm{Mbit} / \mathrm{s}$. With 16 split the maximum of 16 subcarriers will then be needed for each BS (i.e. 2.5G/156.25 Mbit/s). The 256 FFT size in both transmitter and receiver are assumed taking into consideration practical implementation constrains analysed in [4].

CO
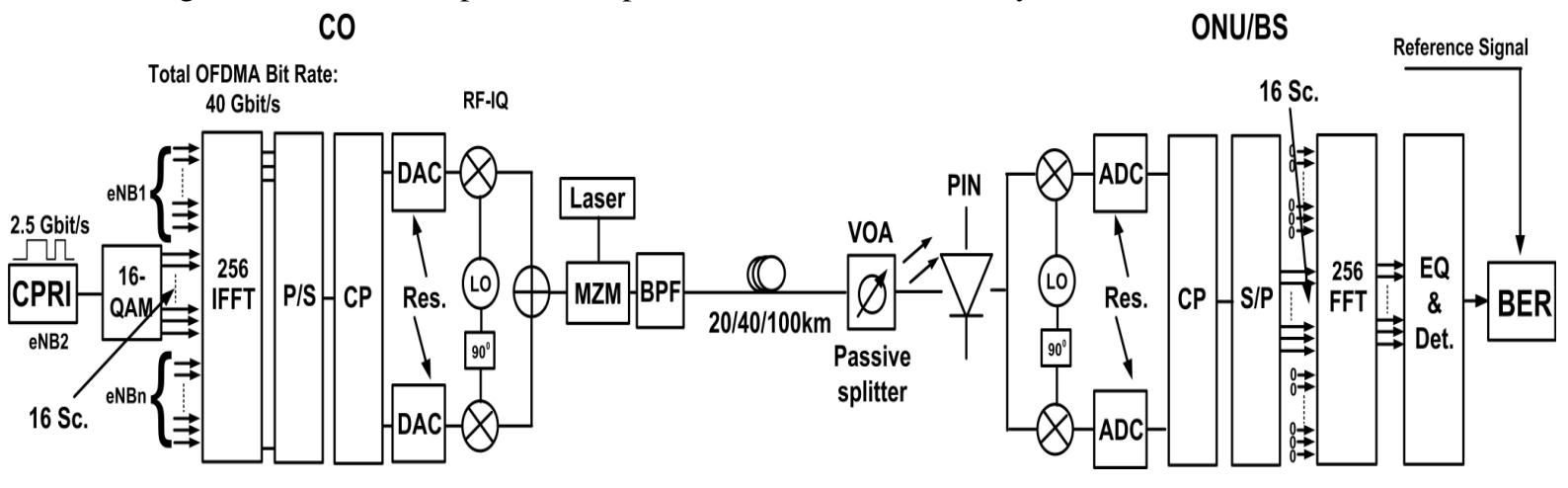

*LO-Local Oscillator; EQ-Equalisation; CP - Cyclic Prefix; VOA: Variable Optical Attenuator

Figure 1. CPRI over OFDMA-PON. 
Table 3. Optical parameters.

\begin{tabular}{|l|l|}
\hline Parameter & Value \\
\hline OFDMA subcarriers (FFT size) & 256 \\
\hline Laser linewidth & $1 \mathrm{MHz}$ \\
\hline Attenuation coefficient on fibre & $0.25 \mathrm{~dB} / \mathrm{km}$ \\
\hline Dispersion parameter & $17 \mathrm{ps} / \mathrm{nm}^{*} \mathrm{~km}$ \\
\hline MZM ER & 20 \\
\hline MZM Chirp factor & -0.176 \\
\hline PIN responsitivity & 0.8 \\
\hline PIN noise (50 GHz) & $21 \mathrm{pA} / \mathrm{Hz}^{\wedge} 1 / 2$ \\
\hline Cyclic Prefix (CP) & $20 \%$ \\
\hline VOA (16 split) & $14 \mathrm{~dB}$ \\
\hline FEC & Not applied \\
\hline
\end{tabular}

Consequently, after the IFFT, cyclic prefix addition and digital to analog conversion, the resulting signal is first up-converted to $30 \mathrm{GHz}$, using an RF-IQ modulator of Fig. 1, and applied to the RF input port of the Mach Zehnder Modulator (MZM) for external laser modulation. The $30 \mathrm{GHz}$ RF frequency was selected in order to introduce a gap between the carrier and desired signal after the photo-detector. In addition, in order to reduce an effect of the chromatic dispersion the optical band pass filter is then applied to filter out a single sideband resulting in an output power of $+3 \mathrm{dBm}$ on the fibre. Finally, the output signal is then transmitted over 20,40 and $100 \mathrm{~km}$ OFDMA-PON links assuming 16 split in the distribution field and the parameters given in Table 3.

At the receiver side, the direct detection, utilising $50 \mathrm{GHz}$ PIN diode, was implemented followed with an electrical RF-IQ down-conversion. Subsequent to the ADC and subcarriers selection for the specific base station, the frequency domain equalisation is then performed after the FFT to estimate amplitude and phase distortion imposed by the optical link on each subcarrier. The equaliser coefficients are estimated based on the four OFDMA training symbols transmitted at the link setup.

\section{RESULTS AND DISCUSSION}

A physical layer simulation test-bed was implemented using Virtual Photonics Inc. (VPI) enriched with MATLAB simulation functionalities to model $2.5 \mathrm{Gbit} / \mathrm{s}$ CPRI transmission over an OFDMA-PON in terms of bit error rates (BER) and ADC/DAC resolution impairments in downstream.

To that extent, the BER versus received power at the PIN diode is plotted in Fig. 2 for various fibre distances and ADC/DAC resolutions. The BER estimation was based on Monte Carlo method (i.e. error counting). The target of 1E-4 was considered for the sensitivity measurements since at this error ratio the application of FEC, such as RS $(255,239)$, could increase the BER to 1E-12 [3]. In order to reduce the simulation time the 50 errors are only counted resulting in total of $2^{19}$ simulated bits.

Consequently, the Fig. 2 (left) demonstrates that for $20 \mathrm{~km}$ fibre and 10 bits ADC/DAC resolution the obtained receiver sensitivity is $-11.5 \mathrm{dBm}$. A small power penalty is observed for 9 bits resolution. The received constellation diagram, displayed as inset in Fig. 2 (left), demonstrates no phase rotation. However, in case of 8 bits resolution the noise floor is reached at BER of 1E-3 signifying that quantisation noise is very high therefore the equaliser at the receiver does not have any effect on the received signal.
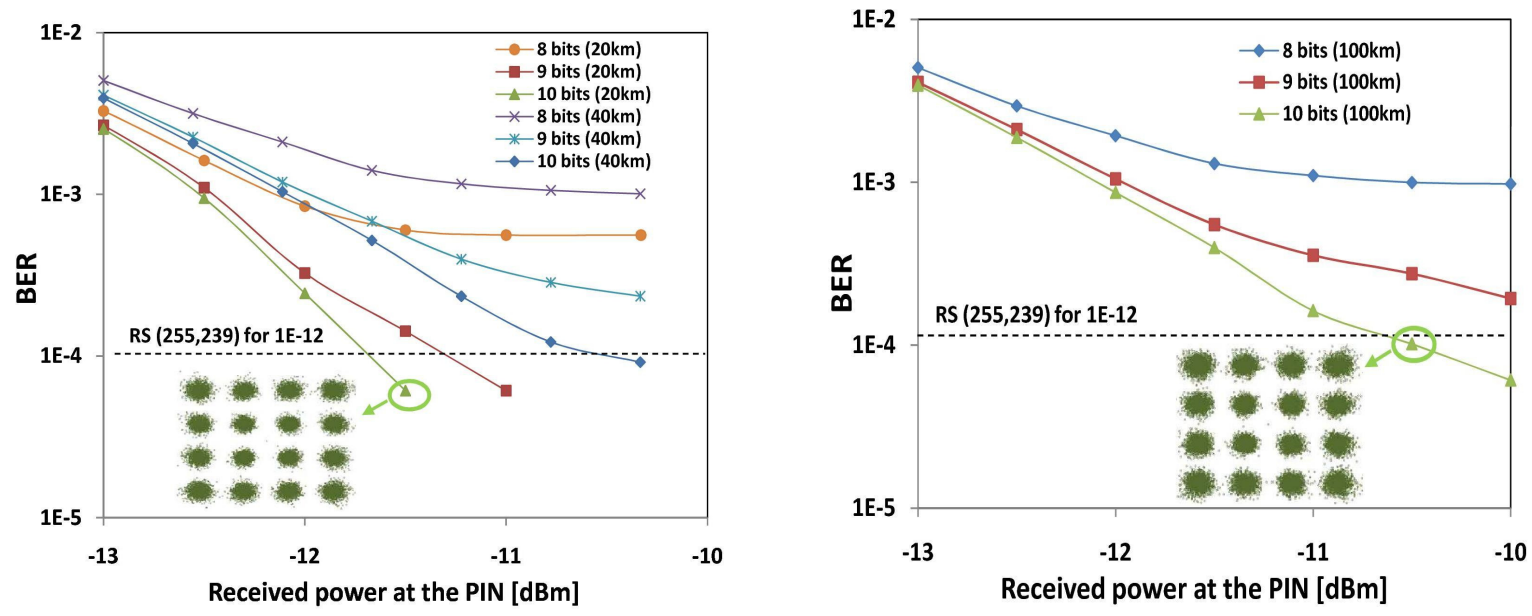

Figure 2. BER versus received power for different DAC/ADC resolutions at 20 and 40 (left) and $100 \mathrm{~km}$ (right). 
In addition, for $40 \mathrm{~km}$ fibre the measured receiver sensitivity for 10 bits resolution was around $-10.5 \mathrm{dBm}$ exhibiting only $1 \mathrm{~dB}$ penalty in comparison to $20 \mathrm{~km}$ transmission. This shows that with the OFDM transmission only simple equaliser is needed in order to compensate for fibre dispersion at long distances. Similarly to $20 \mathrm{~km}$, further penalties were observed for 8 and 9 bits ADC/DAC.

For $100 \mathrm{~km}$ link lengths, shown in Fig. 2 (right), same receiver sensitivity, as with $40 \mathrm{~km}$, of $-10.5 \mathrm{dBm}$ was measured for 10 bits resolution. However, for 8 and 9 bits quantisation noise is significantly degrading the system performance in similar fashion as with $20 \mathrm{~km}$ and $40 \mathrm{~km}$.

Therefore, it can be concluded from the obtained results that the resolution of the ADC/DAC significantly impacts the BER at the receiver and should be carefully considered in practical implementations.

Finally, with 16 split in the distribution field, corresponding to $14 \mathrm{~dB}$ attenuation, the minimum needed optical launch power from the OLT is thus $+7.5 \mathrm{dBm}$ and $+12.5 \mathrm{dBm}$ for $20 \mathrm{~km}$ and $40 \mathrm{~km}$ fibres respectively. These calculations are based on the receiver sensitivity figures of $-11.5 \mathrm{dBm}$ for $20 \mathrm{~km}$ and $-10.5 \mathrm{dBm}$ for $40 \mathrm{~km}$ fibres, taking also into consideration fibre losses. The $100 \mathrm{~km}$ links on the other hand will require an optical amplifier to reach these targets due to high attenuation on the fibre.

It is important to note that the above performance figures were obtained with the low cost solution based on RF-IQ generation at the OLT and direct detection at an ONU. Other signal impairments such as local oscillator drifts, laser linewidth and optical amplifier noise were not considered and could further degrade the performance. Therefore, alternative solutions such as optical IQ generation and coherent detection could be applied instead.

\section{CONCLUSIONS}

This paper presented requirements for next generation wireless backhauling links outlining the key parameters, such as peak data rates and latency that need to be supported. In addition, a DoF converged network architecture is demonstrated featuring CPRI transmission of LTE signals by means of OFDMA techniques over long reach PONs. A low-cost electrical RF-IQ with direct detection approach is implemented. The obtained results demonstrate BER below 1E-4, complying with the CPRI standard with the application of FEC, over total aggregate data rates of $40 \mathrm{Gbit} / \mathrm{s}$ and $100 \mathrm{~km}$ fiber spans. Since the projected network could facilitate the support of multiple operators and multiple technologies within the same infrastructure, it is expected to provide a natural environment for healthy competition among European network and service providers that will revitalize the European market and reinforce its competitiveness.

\section{ACKNOWLEDGEMENTS}

Aspects of this work have been developed to support the European FP7 ACCORDANCE project.

\section{REFERENCES}

[1] S. Ahmadi, "An overview of next-generation mobile WiMAX technology," IEEE Communications Magazine, vol. 47, pp. 84-98, (2009).

[2] D. Astely, E. Dahlman, A. Furuskar, Y. Jading, M. Lindstrom, and S. Parkvall, "LTE: the evolution of mobile broadband - LTE part II: 3GPP release 8," IEEE Communications Magazine, vol. 47, pp. 44-51, (2009).

[3] Common Public Radio Interface (CPRI); Specificaiton v4.1, http://www.cpri.info, 2009

[4] ACCORDANCE: A Converged Copper-Optical-Radio OFDMA-based access Network with high Capacity and Flexibility (Online). Available: http://ict-accordance.eu

[5] M. Milosavljevic, P. Kourtessis, and J. M. Senior, "Transparent wireless transmission over the ACCORDANCE optical/wireless segment," presented at Communication Systems, Networks and Digital Signal Processing (CSNDSP), Newcastle, UK, 2010.

[6] 3GPP TR 25.912 V9.0.0, Feasibility study for evolved Universal Terrestrial Radio Access (UTRA) and Universal Terrestrial Radio Access Network (UTRAN)

[7] 3GPP TS 36.300 V10.3.0, Evolved Universal Terrestrial Radio Access (E-UTRA) and Evolved Universal Terrestrial Radio Access Network (E-UTRAN); Overall description 